\title{
Le tabagisme dans les lycées de la commune VI du district de Bamako
}

\author{
Keïta Sidibé $\mathrm{A}^{1}$, Bah $\mathrm{M}^{1}$, Coulibaly $C A$, Sangaré $\mathrm{Y}^{1}, \mathrm{Maïga} \mathrm{O}^{1}$,Cissé $S A^{1}$, Koné $\mathrm{K}^{1}$, \\ Keïta Dembélé $\mathrm{H}^{1}$, Sangho $\mathrm{F}^{2}$, Sango $\mathrm{HA}^{3}$, Sangho $\mathrm{H}^{1}$.
}

1. Centre de recherche, d'études et de documentation pour la survie de l'enfant (CREDOS), Bamako,

2. Direction de la Pharmacie et du Médicament (DPM) Dar salam Bamako, Mali

3. Faculté de Médecine et d'Odonto-Stomatologie (FMOS), Bamako, Mali

\section{Résumé :}

Le tabagisme reste la principale cause mondiale de décès évitables. II tue près de 6 millions de personnes et entraîne des centaines de milliards de dollars de pertes économiques chaque année dans le monde. Notre étude avait pour but de déterminer la prévalence du tabagisme et d'identifier les facteurs qui l'influencent dans les lycées de la commune $\mathrm{VI}$ du district de Bamako. II s'agissait d'une enquête transversale conduite de mai à juin 2012, et a concerné quatre lycées choisis sur 14 de façon aléatoire.

Etaient inclus dans l'étude, tout élève des deux sexes, âgé de 14 à 23 ans acceptant de répondre aux questions, au total 231 élèves.

II ressort de l'étude que la plupart des fumeurs se trouvaient dans la tranche d'âge de 17-20 ans soit $56,7 \% ; 39,8 \%$ avaient fumé dans le passé et $15,2 \%$ étaient des fumeurs réguliers parmi lesquels $91,4 \%$ étaient du sexe masculin et $8,6 \%$ du sexe féminin. Nous avons trouvé que $51,4 \%$ des fumeurs avaient comme motif d'incitation le plaisir ; $71,4 \%$ avaient essayé d'arrêter de fumer sans pour autant y parvenir ; $81,8 \%$ savaient que le tabac était nocif pour la santé. Nous recommandons d'interdire la publicité et d'intensifier la communication sur le danger du tabac.

Mots clés:Tabagisme- lycée -Bamako-Mali.

\section{Summary:}

Tobacco use remains the leading cause of preventable death. It kills nearly 6 million people and causing hundreds of billions of dollars in economic losses each year in the world. Our study aimed to determine the prevalence of smoking and to identify the factors that influence it in high schools of the municipality VI of Bamako district. This was a cross-sectional survey conducted from May to June 2012 and involved four schools chosen on 14 randomly. Were included in the study, any pupil of the two sexes, old from 14 to 23 years agreeing to answer the questions, on the whole 231 pupils. It is clear from the study that most smokers were in the $17-20$ age bracket is $56.7 \% ; 39.8 \%$ had smoked in the past and $15.2 \%$ were regular smokers of whom $91.4 \%$ were male and $8.6 \%$ female. We found that
$51.4 \%$ of smokers as incentive motive pleasure; $71.4 \%$ had tried to quit smoking without end; $81.8 \%$ knew that smoking was harmful to health. We recommend to ban advertising and intensify communication on the dangers of tobacco.

Keywords : nicotinisme-school-Bamako-Mali.

\section{INTRODUCTION}

Le tabagisme demeure un problème de santé publique dans le monde. En effet environ 1,1 milliard de personnes fument dans le monde et d'ici à 2025 , ce nombre devrait dépasser 1,6 milliard. Si rien n'est fait, d'ici 2030, 10 millions de décès survenant dans le monde seront associés au tabagisme, pour la plupart dans les pays en développement [1]. L'enquête Global Youth Survey (GYTS) réalisée à travers le monde entre 2001 et 2003 chez les élèves de 13 à 15 ans, a permis d'établir une prévalence moyenne de $12 \%$ de fumeurs chez les jeunes africains subsahariens avec des extrêmes allant de 2,9\% en Ethiopie à $28 \%$ au Mali [2 ]. En 2005, l'enquête réalisée dans 4 pays par l'Observatoire du Tabac en Afrique Francophone (OTAF) a montré $22 \%$ de fumeurs chez les élèves du secondaire [2].

Le tabagisme reste la principale cause mondiale de décès évitables. Selon l'OMS, le tabac tue la moitié de ceux qui en consomment. L'épidémie de tabagisme tue près de 6 millions de personnes chaque année. Plus de 5 millions d'entre elles sont des consommateurs ou d'anciens consommateurs, et plus de 600000 , des non-fumeurs involontairement exposés à la fumée. Si aucune mesure n'est prise d'urgence, le nombre annuel de ces décès pourrait atteindre plus de 8 millions d'ici à 2030. Plus de $80 \%$ du milliard de fumeurs dans le monde vivent dans des pays à revenu faible ou intermédiaire. Même si elle baisse dans certains pays à revenu élevé ou à revenu intermédiaire supérieur, la consommation totale de produits du tabac augmente au niveau mondial [3].

Aujourd'hui les méfaits du tabac ne sont plus à prouver car il nuit à la santé des individus dans tous les pays du monde à tous les stades de la vie. Les pathologies les plus connues liées au tabagisme sont entre autres: les cancers de la vessie, de 
l'œsophage, de la cavité buccale, du pharynx, pathologies chroniques des systèmes respiratoire et cardiovasculaire ainsi que l'impact sur le système de reproduction et la mort subite du nourrisson [4].Le tabagisme aggrave la vulnérabilité de ceux qui fument et forme avec la pauvreté un cercle vicieux [5]. L'OMS est engagée dans la lutte contre l'épidémie mondiale de tabagisme. La Conventioncadre de l'OMS pour la lutte antitabac est entrée en vigueur en février 2005. Depuis lors, elle est devenue l'un des traités les plus largement acceptés de l'histoire des Nations Unies, avec 176 parties représentant $88 \%$ de la population mondiale. La Convention-cadre est le principal instrument de l'OMS pour la lutte antitabac et elle marque une étape importante dans la promotion de la santé publique. II s'agit d'un traité fondé sur des bases factuelles qui réaffirme le droit de tout être humain à bénéficier du meilleur état de santé qu'il est capable d'atteindre, qui fournit un cadre juridique pour la coopération sanitaire internationale et fixe des normes exigeantes en matière d'application.

En 2008, l'OMS a lancé une démarche d'un bon rapport coût/efficacité pour accélérer l'application des dispositions de la Convention-cadre de l'OMS. Intitulée MPOWER, celle-ci se décline en une série de «bonnes pratiques» et de «meilleures pratiques " destinées à réduire la consommation de tabac. Chacune d'elles correspond à au moins une disposition de la Convention-cadre de l'OMS pour la lutte antitabac [3].

Une enquête réalisée au Mali en 2008, a montré que $9,5 \%$ des adolescents fumaient [6]. Plusieurs initiatives ont été mises en œuvre pour lutter contre ce fléau, notamment la signature de la Convention Cadre de l'OMS pour la lutte antitabac et l'adoption de la loi 96/041 portant sur la restriction de la publicité et l'usage du tabac au Mali. Les données actuelles étant dépassées, quelle est la situation sur le tabagisme en milieu scolaire au Mali?

Dans le souci d'avoir des données actualisées, nous avons initié ce travail dont l'objectif est d'étudier le tabagisme dans les lycées de la commune VI du district de Bamako.

\section{Matériel et méthodes}

\section{Cadre de l'étude}

L'étude s'est déroulée en commune VI du district de Bamako qui est composée de 10 quartiers, avec une population de 221258 habitants. Elle compte 26 établissements/écoles d'enseignement secondaire général, technique et professionnel dont 14 lycées. Elle comprend un Centre de Santé de Référence et dix Centres de Santé Communautaires [7].

\section{Type et période d'étude}

II s'agissait d'une étude descriptive, transversale à passage unique, réalisée du 28 mai au 13 juin 2012.

\section{Population d'étude}

La population ciblée par cette étude était les élèves âgés de 14 à 23 ans des lycées retenus. Etaient inclus dans l'étude, tout élève des deux sexes, âgé de 14 à 23 ans acceptant de répondre aux questions et exclus de l'étude tout élève des deux sexes fréquentant les lycées retenus dont l'âge est inférieur à 14 ans et supérieur à 23 ans.

Taille de l'échantillon :

La taille de notre échantillon (n) a été calculée selon la formule de Schwartz.

$$
n=(Z \alpha)^{2} \stackrel{p . q}{i^{2}}
$$

Où Za $=$ écart réduit $=1,96$ avec $a=5 \% . \quad p=$ prévalence du tabagisme chez les élèves et étudiants du district de Bamako en 2011 $=18,40 \%$ [3]; $q=1-p$, la probabilité contraire de $p ; \boldsymbol{i}$, la précision attendue des résultats fixée à $5 \%$ dans cette étude.

\section{Echantillonnage}

La commune $\mathrm{Vl}$ a été choisie par tirage aléatoire simple (sur 6 communes). Sur les 14 lycées de la commune $\mathrm{Vl}$, nous avons pris $30 \%$, ce qui correspond à 4 lycées.

Dans chaque établissement, un tirage a été fait au hasard au niveau des classes de $10^{\text {ème }}, 11^{\text {ème }}$ et $12^{\text {ème }}$ année. Nous avons estimé la proportion de chaque classe par rapport à l'effectif de chaque lycée concerné par l'enquête. Le nombre d'élèves à enquêter par classe a été estimé en appliquant la proportion obtenue à la taille de notre échantillon. Tous les élèves volontaires des classes sélectionnées répondant aux critères d'inclusion, ont rempli les questionnaires.

Les variables: les données recueillies ont concerné :

- caractéristiques des élèves: statut tabagique, âge en année, sexe, provenance, niveau d'étude ; - caractéristiques des parents : profession, niveau d'instruction, comportement vis à vis du tabac ;

- attitude et pratique: Statut tabagique: âge d'initiation, durée de consommation, mode d'accès aux cigarettes, quantité de cigarettes fumées par jour, désir d'arrêter, tentative d'arrêter, état de santé du sujet fumeur ;

- niveau de connaissance : sur les risques liés au tabagisme, sur la journée mondiale sans tabac ;

- source d'approvisionnement en tabac (boutique, marchand ambulant) ; 
- connaissance du statut par les parents ;

- influence de l'entourage (frères, amis et autres);

\section{Collecte}

La collecte des données a été faite à partir d'un questionnaire qui a été expliqué aux élèves en salle, et rempli individuellement en présence de l'enseignant et de l'enquêteur. Les questionnaires ont été administrés aux élèves éligibles et les fiches étaient immédiatement récupérées après le remplissage.

\section{Traitement et analyse des données}

Lles données ont été saisies et analysées par le logiciel SPSS 18 version française et la mise en graphique a été effectuée via Microsoft Excel 2007. Des proportions ont été calculées.

\section{Considération éthique}

L'autorisation de l'administration scolaire (académie et proviseurs des lycées concernés) a été obtenue avant l'enquête. Nous avons obtenu le consentement verbal éclairé des élèves après des explications claires sur les objectifs de l'étude. Ils étaient libres d'arrêter à tout moment leur participation à l'enquête. Après le remplissage des questionnaires, la confidentialité des informations recueillies a été garantie.

\section{RESULTATS}

Caractéristiques sociodémographiques des élèves enquêtés :

L'enquête a concerné 231 élèves, répartis entre 4 lycées de la commune VI du district de Bamako.

La tranche d'âge la plus représentée était celle comprise entre 17-20 ans avec 56,7\%. L'âge minimum était de 14 ans et l'âge maximum de 23 ans (Tableau 1).Dans l'échantillon $61,5 \%$ des élèves étaient de sexe masculin avec un sexe ratio de 1,6 en faveur des garçons ; $42 \%$ étaient en 12 ème année (Tableau 1).

Caractéristique des parents des élèves enquêtés Parmi les élèves enquêtés, $90,9 \%$ ont affirmé que leurs pères étaient vivants, pour $22,9 \%$ les pères fumaient de la cigarette et selon $71,7 \%$, ils le faisaient en présence de leurs enfants. II y a 93,9\% des enquêtés qui ont également affirmé que leurs mères étaient vivantes et selon $97,4 \%$, celles-ci ne fumaient pas. Selon les élèves interrogés, $54,5 \%$ ont déclaré qu'ils vivaient avec des fumeurs à la maison et $42,1 \%$ de ces fumeurs présents à la maison étaient des frères.

Selon les lycéens fumeurs, $31,4 \%$ de leurs parents savaient qu'ils fumaient, $22,9 \%$ ne le savaient pas et $45,7 \%$ de ces mêmes lycéens ignoraient si leurs parents étaient conscients de leur tabagisme.
Caractéristique des enseignants des élèves enquêtés

D'après les élèves, la majorité de leurs enseignants fumaient soit $76,6 \% ; 49,1 \%$ le faisaient en classe et $45,8 \%$ dans la cour de l'école. Plus de la moitié des élèves $(60,2 \%)$ n'avaient pas d'antécédents tabagiques.

\section{La prévalence du tabagisme}

-Antécédent tabagique et tabagisme actuel: ॥ ressort de cette étude que 39,8 \% des élèves enquêtés avaient fumé au moins une fois et que $15,2 \%$ étaient des fumeurs réguliers de la cigarette (Tableau 2).

-Sexe : Parmi les fumeurs réguliers, $91,4 \%$ étaient de sexe masculin et $8,6 \%$ de sexe féminin (Tableau 2).

-Durée de consommation et causes du tabagisme: par rapport à la durée de consommation, $48,6 \%$ des fumeurs avaient fumé pendant un an révolu. La motivation première (cause) qui les poussait à fumer était le plaisir avec $51,4 \%$ des cas (Tableau 2).

- Moment de fumer et quantité de cigarette : La majorité des fumeurs interrogés fumaient à tout moment de la journée avec $85,7 \%$ des cas. Près de $14,3 \%$ des fumeurs fumaient 11 à 15 cigarettes par jour, quantité inférieure à $3(31,4 \%), 4$ à 6 cigarettes ljour (31,4\%) (Tableau 2).

- Façon de se procurer les cigarettes et opinion des parents: les fumeurs qui affirmaient avoir acheté eux-mêmes leurs cigarettes étaient de $80 \%$; selon $31,4 \%$, leurs parents savaient qu'ils fumaient, $22,9 \%$ ne le savaient pas et $45,7 \%$ de ces mêmes lycéens ignoraient si leurs parents étaient conscients de leur tabagisme. Les fumeurs interrogés rapportaient que $81,8 \%$ de leurs parents pensaient que fumer est une mauvaise chose et 9,1\% étaient indifférents (Tableau 2).

\section{Connaissance des élèves sur le tabagisme}

- Connaissance de la nocivité sur la santé et les maladies :Parmi les élèves enquêtés, $81,8 \%$ affirmaient connaître que le tabac entraîne des maladies et parmi ceux-ci, $33,3 \%$ faisaient le lien entre le tabagisme et le cancer des poumons (Tableau 3).

- Connaissance de la journée mondiale sans tabac et de la loi sur la vente et la consommation du tabac: Concernant la journée mondiale sans tabac, $79,7 \%$ des lycéens ignoraient son existence. A propos de la loi sur la vente et la consommation du tabac au Mali, 74,5\% des lycéens ignoraient son existence (Tableau 3).

Enseignement sur les dangers du tabac à l'école : il y a 53,7\% des élèves qui affirmaient 
avoir reçu un enseignement sur les dangers du tabac à l'école (Tableau 3).

\section{Lutte antitabac}

Interdiction de fumer à l'école, dans la salle de réunion, dans le transport en commun et au bureau: dans notre étude, $74,5 \%$, des lycéens trouvaient normale l'interdiction de fumer à l'école et dans la salle de réunion, $68,4 \%$ dans le transport en commun et $66,2 \%$ au bureau.

Publicité du tabac : chez ces mêmes élèves, $81,8 \%$ estimaient que ce n'est pas bien de faire la publicité du tabac. Parmi les lycéens fumeurs, $71,4 \%$ avaient tenté d'arrêter de fumer. Le motif d'arrêt pour des raisons de santé était le plus évoqué avec $92 \%$ des cas. Les fumeurs qui projetaient d'arrêter un jour la consommation du tabac étaient de $60 \%$.

\section{Tentative d'arrêt du tabac et causes :}

Les résultats de l'enquête montrent que $71,4 \%$ des fumeurs affirmaient avoir essayé d'arrêter de fumer sans pour autant y parvenir. Les raisons de santé constituaient la première motivation des jeunes à vouloir arrêter le tabac $(92 \%)$.

\section{DISCUSSION}

\section{Caractéristiques sociodémographiques}

Age :

La tranche d'âge de 17-20 ans était prédominante avec $56,7 \%$ des cas. Au Mali, la tranche d'âge la plus fréquente était comprise entre 15 et 20 ans soit $83,1 \%$ [8]. On constate que l'âge d'initiation au tabac a augmenté de 15 à 17 ans. Ceci pourrait s'expliquer par les différentes sensibilisations menées sur le tabagisme.

D'autres études réalisées en Afrique ont montré une variation de l'âge des scolaires. Ainsi Netti au gabon [9] a trouvé que les enfants âgés de 20-22 ans étaient majoritaires avec $42,9 \%$; $\mathrm{A}$ Madagascar Befinoana a rapporté $48 \%$ pour les 16 18 ans [10]. F. Koueta au Burkina Faso a trouvé $66 \%$ pour les $15-19$ ans [11].

\section{Sexe :}

Plus de la moitié des élèves $(61,5 \%)$ étaient de sexe masculin avec un sexe ratio de 1,6 en faveur des hommes.

\section{Caractéristiques des parents des élèves enquêtes}

La grande majorité soit 90,9 \% des élèves interrogées, affirmaient que leurs pères étaient vivants ; $22,9 \%$ étaient fumeurs de cigarette et $71,7 \%$ le faisaient en présence de leurs enfants. Au Mali, $55 \%$ des pères des jeunes fumeurs fumaient et parmi ces pères, $75,25 \%$ fumaient en présence de leurs enfants [8]. Les résultats du GYTS $2008 \mathrm{du}$
Mali ont montré que $21,7 \%$ des élèves ont l'un ou les deux parents qui fument [6].

Selon une étude réalisée en Côte d'lvoire, l'incidence du tabagisme parental représentait $26,5 \%$ [12].

Dans notre étude, la présence d'autres fumeurs à la maison a été évoquée par $54,5 \%$ des élèves interrogés et les frères ont été cités par $42,1 \%$. Au Burkina, les trois quarts des élèves fumeurs ont déclaré avoir au moins un membre de la famille qui fume. II s'agissait du père $(70 \%)$, du frère $(20 \%)$, du père et du frère (10\%) [11]. Au Sénégal, $29 \%$ des fumeurs avaient un ami fumeur, un parent fumeur pour 24\% [13].

Selon les lycéens fumeurs dans notre étude, $31,4 \%$ de leurs parents savaient qu'ils fumaient, $22,9 \%$ ne le savaient pas et $45,7 \%$ de ces mêmes lycéens ignoraient si leurs parents étaient conscients de leur tabagisme. Selon une étude réalisée en Côte d'Ivoire, $13,8 \%$ des parents savaient que leurs enfants fumaient [12].

Dans notre étude, les fumeurs interrogés rapportaient que $81,8 \%$ de leurs parents pensaient que fumer est une mauvaise chose et $9,1 \%$ étaient indifférents. Au Burkina, $71,3 \%$ ont affirmé qu'ils ont été réprimandés par les parents. Cependant $6 \%$ ont été encouragés dans leur tabagisme par l'attribution de l'argent du tabac, 22,7\% des parents étaient indifférents [11].

\section{Caractéristiques des enseignants des élèves enquêtes}

L'enquête menée auprès des élèves a révélé que la majorité de leurs enseignants $(76,6 \%)$ fumaient. IIs le faisaient dans l'environnement scolaire et précisément dans la classe $(49,1 \%)$ et dans la cour de l'école (45,8\%). Au Sénégal, 100\% des élèves fumeurs avaient au moins un professeur fumeur [13]. En Côte d'Ivoire, plusieurs élèves $(37,7 \%)$ ont affirmé que leurs enseignants fumaient au sein de l'école en leur présence [12].

Ces résultats mettent en exergue le comportement négatif des enseignants qui devraient plutôt enseigner les bonnes pratiques aux élèves et servir de modèles à leurs yeux. II faut organiser des séances de sensibilisation sur les dangers du tabac à l'endroit des enseignants et appliquer la loi 96/041 portant sur la restriction, la publicité et l'usage du tabac au Mali.

\section{Tabagisme chez les élèves - Antécédents tabagiques}

Dans notre étude, il ressort que 39,8 \% avaient fumé au moins une fois. Aux Comores [14], il ressort que $26,5 \%$ des élèves âgés de $13-15$ ans enquêtés ont déjà fumé la cigarette. 
- Tabagisme actuel (La prévalence du tabagisme) :

II ressort de l'étude, que 15,2 \%des élèves étaient des fumeurs réguliers de cigarettes. Des études similaires menées en Afrique ont trouvé des prévalences de $13 \%$ [13] et plus de 15,9\% [12].

En Tunisie, Afifa et al ont trouvé que la fréquence du tabagisme chez les collégiens était de $26 \%$ [15].

Sexe (fumeurs):

Parmi les fumeurs réguliers, les garçons étaient majoritaires avec $91,4 \%$ contre $8,6 \%$ de filles. Au Burkina [11], la grande majorité $(96 \%)$ des élèves fumeurs étaient de sexe masculin.

\section{Durée de consommation et causes du tabagisme :}

Dans la présente étude, par rapport à la durée de consommation, $48,6 \%$ des fumeurs avaient fumé pendant un an révolu. Au Mali, Diallo SI et al [8] ont trouvé que $84,1 \%$ des jeunes fumeurs fumaient depuis plus d'un an. Kodio au Mali a trouvé une durée de consommation chez les fumeurs supérieure à 1 mois (52\%) [16].

La principale motivation qui les incitait à fumer était le plaisir avec 51,4\% des cas. Au Burkina, Koueta a trouvé que près de la moitié soit $46 \%$ des élèves avait fumé par imitation de l'entourage [11]. En Tunisie, parmi les motifs les plus évoqués, on retrouve en plus du caractère apaisant de la cigarette (47\%) le plaisir de fumer (35\%) [15]. Pour Kodio [16], les principales motivations étaient : la promiscuité avec $63 \%$ suivie du plaisir (22\%) et le snobisme avec $13 \%$.

\section{Moment de fumer}

Dans cette étude, le moment de fumer dans la journée n'était pas défini. La majorité des fumeurs interrogés fumaient à tout moment de la journée avec $85,7 \%$ des cas. Le fait de fumer à tout moment de la journée a été également rapporté par Kodio [16] mais à des proportions inférieures (61\%).

\section{Quantité de cigarette}

Plus de la moitié $(63 \%)$ des fumeurs fumaient 1 à 6 cigarettes par jour et $14,3 \%$ de 11 à 15 cigarettes. Ce résultat est similaire à celui de Kodio au Mali qui avait trouvé que $63 \%$ des fumeurs fumaient 1 à 5 cigarettes par jour [16].Au Burkina [11], a rapporté que le nombre de cigarettes consommés par jour variait de 2 à 20 cigarettes.

Façon de se procurer les cigarettes

La majorité $(80 \%)$ des fumeurs avaient acheté euxmêmes leurs cigarettes. Selon Kodio, parmi les élèves fumeurs, $67,4 \%$ affirmaient avoir acheté euxmêmes leurs cigarettes [16].
Pour l'acquisition de la cigarette, $F$ Koueta [11] et Kouassi [12] ont trouvé respectivement $85,2 \%$ et $62,7 \%$ pour l'argent de poche.

Notre étude a montré que $31,4 \%$ des parents des lycéens fumeurs connaissaient le statut tabagique de leurs enfants, $22,9 \%$ ne le savaient pas. Selon Kodio [16]. $37 \%$ des parents des élèves fumeurs savaient qu'ils fumaient tandis que $63 \%$ l'ignoraient. En Tunisie, 18\% des fumeurs ont eu l'autorisation de leurs parents [15].

La grande majorité des fumeurs interrogés $(81,8 \%)$ rapportaient que leurs parents n'étaient pas favorables à la consommation du tabac et $9,1 \%$ étaient indifférents. Selon Kodio il y avait $76 \%$ des parents qui pensaient que le tabagisme des enfants est une mauvaise chose [16].

\section{Connaissance des élèves sur le tabagisme : Connaissance de la nocivité sur la santé :}

La majorité des élèves interrogés $(81,8 \%)$ savait que le tabac était nocif pour la santé. Selon une étude faite au Sénégal, en 2011 sur le tabagisme en milieu scolaire dakarois, la majorité des élèves $(86 \%)$ ont cité les méfaits du tabac [13].

En côte d'lvoire, moins des $2 / 3$ des élèves connaissaient les conséquences du tabac [12].

\section{Connaissance maladies :}

Dans notre étude, les maladies connues étaient: Cancer des poumons $(33,3 \%)$, maladies des poumons $(49,8 \%)$, maladies du cœur $(15,9 \%)$, cancer du foie (1\%). Au Sénégal, ils ont cité surtout : Cancer (42\%),pneumopathie (40\%), cardiopathie (21\%) [13].

Selon B.A. Kouassi et al, les élèves ont cité les pathologies pulmonaires $(62,9 \%)$ et plus précisément du cancer broncho-pulmonaire $(63 \%$ des pathologies pulmonaires) [12].

Au Burkina, la grande majorité $(82,4 \%)$ connaissait la nocivité du tabac sur la santé. La toux $(83,8 \%)$ et le cancer du poumon $(79,8 \%)$ ont été les maladies causées par le tabac les plus citées par les élèves [11].

\section{Enseignement des dangers du tabac à l'école}

Plus de la moitié $(53,7 \%)$ des élèves affirmaient avoir reçu un enseignement sur les effets nocifs du tabac. En 2008 , près de $46 \%$ des élèves âgés entre 13 et 15 ans ont reçu un enseignement à l'école sur les effets néfastes de la cigarette contre $36 \%$ en 2001[17].

Selon Kodio, parmi les élèves enquêtés, 61,3\% déclaraient ne pas recevoir d'enseignement à l'école sur les dangers du tabac [16]. Aux Comores, $36,5 \%$ des élèves enquêtés ont été sensibilisés à l'école sur les dangers du tabac [14]. En RCA, 
$40,8 \%$ des élèves ont été enseignés sur le tabagisme [17].

\section{Connaissance de l'existence de la journée sans tabac \\ Concernant la Journée Mondiale sans Tabac, $79,7 \%$ des lycéens ignoraient son existence. Selon Kodio, $78 \%$ ignoraient l'existence de la journée mondiale sans tabac [16].}

Connaissance de l'existence de la loi antitabac au Mali (Connaissances de la loi sur la vente et la consommation du tabac au Mali)

Concernant la journée mondiale sans tabac, 79,7\% des lycéens ignoraient son existence. A propos de la loi sur la vente et la consommation du tabac au Mali, 74,5\% des lycéens ignoraient son existence. Kodio a trouvé que $76,5 \%$ ignoraient l'existence de la loi antitabac [16].

\section{Lutte antitabac}

Avis des élèves sur les actions anti-tabacs - Interdiction de fumer à l'école et en salle de réunion

Près des $3 / 4$ des lycéens de l'étude trouvaient normale l'interdiction de fumer à l'école et dans les salles de réunion. Selon Kodio, la quasi-totalité des élèves trouvait normale l'interdiction de fumer à l'école et dans les salles de réunion avec 96\%[16].

- Interdiction de fumer dans les transports en commun

L'interdiction de fumer dans les transports en commun était perçue normale par $68,4 \%$ des élèves.

Kodio dans son étude avait trouvé que 95,7\% des élèves pensaient normale l'interdiction de fumer dans les transports en commun [16]. Une étude réalisée au Mali, avait trouvé que $86,9 \%$ sont favorables à l'interdiction de fumer dans les endroits publics [17].

Au Sénégal, parmi les solutions proposées pour lutter contre le tabac, $18 \%$ était pour l'interdiction de fumer dans les lieux publics [13].

\section{- Interdiction de fumer au bureau}

Dans notre étude, ceux qui trouvaient normale l'interdiction de fumer au bureau étaient de 66,2 \%. Kodio a trouvé que $95,7 \%$ des élèves pensaient normale l'interdiction de fumer au bureau [16 ].

\section{Publicité du tabac}

Parmi les élèves enquêtés, $81,8 \%$ étaient contre la publicité du tabac. Selon Kodio [16]. la publicité du tabac était perçue comme une mauvaise chose par 76,2\%. Au Sénégal, parmi les solutions proposées pour lutter contre le tabac, $18 \%$ était pour l'arrêt de la publicité [13].

Tentative d'arrêt du tabac
II ressort de notre étude, que $71,4 \%$ des fumeurs affirmaient avoir essayé d'arrêter de fumer sans pour autant y parvenir. Notre résultat est inférieur à celui évoqué par Faye au Sénégal qui était de $88 \%$ [13].

En Tunisie, une étude a rapporté que 23\% des élèves fumeurs ont déjà essayé d'arrêter de fumer sans y parvenir [15]. Selon l'enquête GYTS 2008, $73 \%$ des fumeurs réguliers souhaiteraient arrêter de fumer maintenant [6]. Selon Kodio, parmi les élèves enquêtés, $61 \%$ ont essayé d'arrêter de fumer [16].

\section{Cause de la tentative d'arrêt}

Dans la présente étude, les raisons de santé constituaient la première motivation des jeunes à vouloir arrêter le tabac (92\%). En Tunisie, Afifa et al ont trouvé que la motivation réside dans le caractère dangereux pour la santé (94\%) et dans le coût du tabagisme (44\%) [15]. Selon Kodio, la dangerosité du tabac pour la santé était la motivation première de la tentative d'arrêt du tabac avec $65 \%$ [16].

\section{CONCLUSION}

La prévalence globale du tabagisme dans les lycées de la commune VI du district de Bamako était de $15,2 \%$. Cette fréquence variait selon le sexe soit $91,4 \%$ des garçons contre $8,6 \%$ des filles. La plupart des fumeurs se trouvaient dans la tranche d'âge $17-20$ ans soit $56,7 \%$ des cas. Le motif d'incitation le plus souvent évoqué était le plaisir $51,4 \%$. La majorité des élèves interrogés soit $81,8 \%$ savaient que le tabac était nocif pour la santé.

Le niveau relativement élevé de la prévalence des fumeurs chez ces élèves interpelle les autorités à mettre en place des stratégies plus efficientes dans la lutte antitabac. D'autres études doivent être programmées pour surveiller l'évolution de cette épidémie et évaluer l'efficacité des stratégies en place pour la réduction des facteurs qui influencent sa propagation.

\section{REFERENCE}

1- Lamarre M-C, Ratte S,Hirsch A. La crise croissante $\mathrm{du}$ tabagisme en Afrique francophone. Promotion \& Education. Revue Internationale de Promotion de la Santé et d'éducation pour la Santé. $2005 ; 4$ : p76.

2. Mor Ndiaye, Mamadou Lamine Sow. Le tabagisme en Afrique Subsaharienne: un nouveau défi ?. Rev Pneumol Trop. $2008 ; 10$ : 33-39.

3-Organisation Mondiale de la Santé. Tabagisme. Aide-mémoire $\mathrm{N}^{\circ} 339$. Genève; 2013 [cité le 15 octobre 2014]. Disponible à : 
http://www.who.int/mediacentre/factsheets/fs339 |frl

4-Organisation Mondiale de la Santé. Epidémie mondiale du tabagisme. Rapport. Genève; 2013.

5-Association de Lutte contre le tabac, l'alcool et les stupéfiants au Mali (ALUTAS). Le tabac et la pauvreté au Mali. Rapport d'enquête. 2010.

6-Organisation Mondiale de la Santé, Ministère de la santé (Mali). Enquête sur le tabac chez les adolescents en milieu scolaire au Mali. Rapport Mali. 2008.

7-Ministère de l'administration territorial et des collectivités locales (Mali). Etude monographique de la commune VI du district de Bamako. 2004.

8-Diallo SI, Tchuindem SI, M'Baye OI, Sissoko BI, Kayantao DI, Dao S.Tabagisme chez les Lycéens dans le District de Bamako. Mali Medical, 2004 ; XIX (3\&4) : 36-9.

9-Nzouzi N, Piette D.Tabagisme en milieu scolaire secondaire du Gabon Prévalence et facteurs psychosociaux associés. Cahiers Santé. 2007 ; 17(3) : 159-65.

10-Befinoana M, Razanamihaja N. (2011) Tabagisme et facteurs associés chez les adolescents scolarisés à Madagascar. Rev Santé Publique, 2007 ; 23 (6) 1-11 : 465-474.

11-Koueta $F$, Dao L, Yé D, Koura $M$, Sawadogo.Facteurs favorisant le tabagisme des élèves à Ouagadougou (Burkina Faso). Rev Mal Respir, 2009 ; 26 : 291-7.

12-Kouassi B A, Horo K, Nigue L, Kassi O, Ahui B J $M$, Koffi N.Tabagisme en milieu scolaire dans la commune de Cocody á Abidjan. Rev pneumologie clinique. 2007 ; 63 (1) : 35-9.

13-Faye A, Seck I, Seye Ndiaye A C, Ndiaye P, Dia Tal A. Aspects épidémiologiques du tabagisme en milieu scolaire dakarois, au Sénégal. Med Afr Noire. 2011 ; (5807) : 363-369.

14-Vice-présidence, chargée de la santé, de la solidarité et de la promotion du genre (Comores). Enquête sur le tabac chez les adolescents en milieu scolaire aux Comores. Rapport. 2008.

15-Koubaa A A, Chibani M, Abed N B, Dahmen H, Ouerfelli $N$, Maabouj M T et al. Le tabagisme chez les collégiens de la région de Zaghouan. Tunisie Médicale. 2009 ; 87 (08) : 527-531.

16-Sangho H, Keita A S, Kodio A, Tayeb M M, DembeleKeita $\mathrm{H}$, Cissé $\mathrm{M} \mathrm{O}$ et al. Tabagisme en milieu scolaire dans une commune de Bamako au Mali. Mali Med. 2014 ; XXIX (1) :369.

17-Kamalo C. Enquête globale sur le tabagisme chez les jeunes de 13 à 15 ans en milieu scolaire à Bangui république centrafricaine. 2008.

Tableau 1 : Caractéristiques sociodémographiques des lycéens de la commune VI du district de Bamako en juin 2012

\begin{tabular}{lcc}
\hline Caractéristiques sociodémographiques & Fréquence & Pourcentage \\
\hline Age $(\mathbf{n}=\mathbf{2 3 1})$ & 79 & \\
$14-16$ & 131 & 34,2 \\
$17-20$ & 21 & $\mathbf{5 6 , 7}$ \\
$21-23$ & & 9,1 \\
Sexe $(\mathrm{n}=231)$ & 142 & 61,5 \\
Masculin & 89 & 38,5 \\
Féminin & & \\
\hline
\end{tabular}


Tableau 2 : Comportement tabagique des lycéens de la commune VI de Bamako en Juin 2012

\begin{tabular}{|c|c|c|}
\hline Comportement tabagique & Fréquence & Pourcentage \\
\hline \multicolumn{3}{|c|}{ Antécédent tabagique ( $\mathrm{n}=231$ ) } \\
\hline Non & 139 & 60,2 \\
\hline Oui & 92 & 39,8 \\
\hline \multicolumn{3}{|l|}{ Tabagique actuel $(n=231)$} \\
\hline Non & 196 & 84,8 \\
\hline Oui & 35 & 15,2 \\
\hline \multicolumn{3}{|l|}{ Sexe $(n=35)$} \\
\hline Masculin & 32 & 91,4 \\
\hline Féminin & 3 & 8,6 \\
\hline \multicolumn{3}{|c|}{ Durée de consommation ( $n=35$ ) } \\
\hline 1 an & 17 & 48,6 \\
\hline 4ans & 7 & 20 \\
\hline 2 ans & 6 & 17,1 \\
\hline 3 ans & 4 & 11,4 \\
\hline 5 ans & 1 & 2,9 \\
\hline \multicolumn{3}{|c|}{ Quantité de cigarette fumée $(n=35)$} \\
\hline$<3$ & 11 & 31,4 \\
\hline $4-6$ & 11 & 31,4 \\
\hline $7-10$ & 8 & 22,9 \\
\hline $11-15$ & 5 & 14,3 \\
\hline \multicolumn{3}{|c|}{ Façon de se procurer de la cigarette } \\
\hline J'en achète & 28 & 80 \\
\hline On m'en offre & 7 & 20 \\
\hline
\end{tabular}

Tableau 3 : Connaissance des élèves sur les dangers du tabagisme et enseignement des dangers du tabac à l'école

\begin{tabular}{lcc}
\hline $\begin{array}{l}\text { Connaissance sur les dangers du tabagisme et } \\
\text { enseignement des dangers du tabac à l'école }\end{array}$ & Fréquence & Pourcentage \\
\hline Connaissance des maladies du Tabac (n=231) & & \\
Oui & 189 & $\mathbf{8 1 , 8}$ \\
Non & 42 & 18,2 \\
Maladies connues (n=231) & & \\
Cancer des poumons & 63 & 33,3 \\
Maladies des poumons & 47 & 24,9 \\
Toux & 47 & 24,9 \\
Maladies du cœur & 30 & 15,9 \\
Cancer du foie & 2 & 1 \\
\hline Connaissance de l'existence de la loi antitabac au Mali (n=231) & \\
Non & 172 & 74,5 \\
Oui & 59 & 25,5 \\
Connaissance de l'existence de la journée sans & & \\
tabac (n=231) & & \\
Non & 184 & 79,7 \\
Oui & 47 & 20,3 \\
Enseignement des dangers du tabac à l'école (n=231) & & \\
Oui & 124 & $\mathbf{5 3 , 7}$ \\
Non & 107 & 46,3 \\
\hline
\end{tabular}

